\title{
Expression contrôlée de protéines exogènes létales dans les cellules eucaryotes: "tout est dans la tet"
}

La transfection stable d'un gène ectopique au sein des cellules d'une lignée permet d'observer directement son effet sur le comportement de ces cellules modifiées par rapport aux cellules témoins cultivées en parallèle. Cette technique est couramment utilisée pour étudier le phénotype d'un gène ou d'un gène modifié à effet dominant. Toutefois, elle comporte des limites. En effet, elle ne permet pas d'étudier l'effet induit par des protéines létales ou inhibant la prolifération: les cellules exprimant la protéine exogène ne peuvent se diviser et la phase de sélection au cours de laquelle sont éliminées toutes les cellules ne portant pas le gène d'intérêt et le gène de sélection associé ne peut s'accomplir. De même, l'étape ultérieure de clonage qui consiste à reconstituer à partir d'une cellule isolée une colonie complète de plusieurs milliers de cellules identiques ne peut avoir lieu. La solution à ce problème consiste à réprimer l'expression du gène transfecté durant ces étapes d'obtention de la colonie de cellules modifiées. L'expression est alors induite au moment choisi.

Le système tétracycline mis au point par Gossen et Bujard [1] consiste à cloner en deux étapes successives (1) le gène codant pour la composante transactivatrice, (2) le gène d'intérêt placé sous la dépendance d'un «super» promoteur viral. Ce promoteur est lui-même couplé à un élément de régulation, dérivé d'un système procaryote, capable de fixer la protéine transactivatrice. La régulation de l'activité de ce système d'expression se fait par l'ajout dans le milieu de culture de l'antibiotique tétracycline qui, en se fixant au complexe de transactivation, en inhibe l'interaction avec le promoteur. Ce système inductible présente la caractéristique essentielle d'être sans effet qui n'est pas le cas pour d'autres systèmes inductibles utilisant des promoteurs activés par des métaux lourds, des hormones, ou par choc thermique pour lesquels on observe des effets secondaires, mais également un niveau basal d'expression élevé.

Cette nouvelle technique est aujourd'hui utilisée avec succès, et on dénombre chaque année un nombre croissant de publications y référant. Le brevet déposé par les auteurs a, quant à lui, été racheté par une grande compagnie pharmaceutique.

Deux résultats récemment obtenus en utilisant ce système correspondent à l'étude de deux gènes qu'il est impossible de cloner de manière stable dans des cellules, puisque le premier induit l'arrêt du cycle cellulaire, et le second, la mort cellulaire programmée.

Niculescu et al. (La Jolla, CA, USA) [2], étudiant le rôle joué par la protéine p21 wafl/cip1 , un inhibiteur des kinases dépendantes des cyclines [3], comme régulateur négatif de la progression du cycle cellulaire ont utilisé le système tétracycline pour montrer que la surexpression de p21 peut induire non seulement un arrêt du cycle cellulaire à la fin de la phase $\mathrm{G} 1 / \mathrm{S}$, mais également à l'interphase G2/M.

Xiang et al. (Saint-Louis, MO, USA) [4] ont mis au point un système de type tétracycline permettant le contrôle de la synthèse de la protéine Bax au sein de cellules lymphoblastiques: des signes d'apoptose apparaissent 10 heures après l'induction de l'expression du gène $B A X$. L'apoptose est donc induite par la seule surexpression de cette protéine, contrairement à ce qui avait été démontré au préalable dans le même laboratoire, sur des cellules transfectées de manière stable, dans lesquelles l'apoptose n'était observée que lorsque les cellules recevaient un stress supplémentaire [5].

Ces deux exemples illustrent clairement les avantages offerts par ce sys- tème cellulaire. Certaines options y ont en outre été ajoutées. Par exemple, les inventeurs du procédé originel ont mis au point un système dérivé dans lequel l'induction de la protéine exogène se fait par l'ajout de tétracycline et non par son retrait (ce qui évite d'avoir à rajouter de la tétracycline quotidiennement dans le milieu de culture). Il existe également des systèmes permettant l'expression simultanée de deux gènes d'intérêt, et d'autres capables de coexprimer les éléments de régulation et le gène étudié. Ces derniers sont portés par un seul vecteur de type rétroviral produit par des cellules eucaryotes infectées. Enfin, des sites Internet spécialisés permettent d'obtenir des informations complémentaires. Par exemple, le professeur Bujard met à disposition, sur le site de son laboratoire (http://www.zmbh.uni-heidelberg.de/bujard/homepage.html), certains protocoles expérimentaux ainsi que les cartes de restriction des plasmides utilisés.

Un autre site, créé sous l'initiative de Sandra Schmid: (http://www.elsevier.nl/inca/homepage/sab/ito/tet main.htm), permet la mise en contact des chercheurs proposant de donner les lignées qu'ils ont produites avec ceux qui en auraient l'utilité.

T.R.

1. Gossen M, Bujard H. Tight control of gene expression in mammalian cells by tetracycline-responsive promoters. Proc Natl Acad Sci USA 1992; 89: 5547-51.

2. Niculescu B, Chen X, Smeets M, Hengst L, Prives C, Reed SI. Effects of p21 ${ }^{\text {Cip1/wafl }}$ at both G1/S and G2/M cell cycle transitions. Mol Cell Biol 1998; 18: 629-43.

3. Cayrol C, Ducommun B. Interaction entre l'inhibiteur des kinases dépendantes des cyclines p21 et le PCNA: un lien entre un cycle cellulaire, la réplication et la réparation de l'ADN. Med Sci $1997 ; 13: 1259-65$.

4. Xiang J, Chao DT, Korshmeier J. BAX induced cell death may not require interleukin $1 \beta$-converting enzyme-like proteases. Proc Natl Acad Sci USA 1996; 93: 14559-63.

5. Oltvai Z, Milliman C, Korshmeyer J. Bcl-2 heterodimerizes in vivo with a conserved homolog, Bax, that accelerates programmed cell death. Cell 1993; 74: 609-19. 